\title{
On the physical nature of remote precursors (Magma and volatile transport as a possible key issue of the earthquake preparation process and appearance of remote precursors)
}

\author{
Igor I. Rokityansky \\ Institute of Magnetism, POB-338/7, Kiev-146, Ukraine
}

\begin{abstract}
Stress accumulation in the focal zone of an impending earthquake is traditionally considered as a primary cause of all precursory phenomena. Such an approach meets difficulties in the explanation of remote precursors: volatile outcome, level and composition of ground water, variations of electric and magnetic fields, EM-emission, atmospheric luminosity, variations of ionospheric parameters, cloud lineaments appearance etc. A change in paradigm is required to proceed in explanation of the remote precursors and their application to the prediction of subsequent earthquake. Some elements of a new paradigm of the earthquake preparation process are proposed based on recently published results: 1 . Earthquake preparation and formation of precursors take place in a much larger volume than the source focal volume of subsequent earthquake. 2. Volatile transport and release from the deep interior is one of basic factors of earthquake preparation and precursors formation processes. 3. Magma emplacement and transport along Earth's crust can be a cause of some earthquakes and their precursors. Terrestrial data of intrusive geology and modern volcanology as well as satellite survey on Venus revealed abundant radiating dyke swarms with radius up to $2000 \mathrm{~km}$. It means that lateral magma emplacement was and continue to be widely spread in the Earth's crust. Magma emplacement can cause earthquakes including strong ones, which is well known for volcanic environment. Usually volcanic earthquakes are not very strong because excessive pressure can be released by either eruption or Earth's surface inflation. In deeper strata, magma emplacement can meet a region where large scale stress accumulation can occur which can result in a strong earthquake. Dissipation of energy from hot magma to cold host rocks put limitation on the magma emplacement duration, which typically lasts days or weeks according to model estimations. Short term precursors can be generated in any place of magma emplacement path, even far away from the source focal volume of impending earthquake. It can explain some enigmatic peculiarities of observed precursors, for example, sensitivity and selectivity of seismic electric signals (SES) in Greece.
\end{abstract}

Keywords: earthquake preparation process; source focal volume, precursor; dyke swarm; magma emplacement; seismic electric signal

\section{Introduction}

According to the commonly accepted idea, an earthquake (EQ) preparation consists of gradual accumulation of stress in the source volume. The stress accumulation creates a set of observable effects: vertical and horizontal movements of the Earth's crust, change in the level 
and composition of ground water, volatile outcome, variations of electric and magnetic fields and so on. These effects can be predicted and evaluated using physical theory. The theory yields strong attenuation of the effects of EQ preparation with increase of the distance from the source volume. Nevertheless, EQ-precursory effects are observed not only near the epicentral zone (where they are expected according to the theory) but also far from it. Furthermore, there are unexpected precursory effects: luminosity of atmosphere, EM-emission, changes of ionospheric parameters, cloud lineaments appearance etc. (Hayakawa, 1999, Hayakawa and Fujinawa, 1994, Morozova, 1993). All these phenomena, which are difficult for scientific explanation, can be considered as anomalous. Absence of an appropriate physical theory of earthquakes and their precursors is one of the causes of very poor success in EQ predictions. The situation gives rise to the skeptical idea of "unpredictability of EQ" (Geller, 1997). The author considers that strong EQ can be studied, understood and predicted in reasonable limits. And nature gives us an evidence of predictability of EQ: some living beings have an unambiguous presentiment of $\mathrm{EQ}$, for example, serpents even being in winter hibernation wake up several hours before EQ and try to run away from epicentral zone (Sharigin et al., 1995a, b). It means that reliable information about an impending strong EQ does exist and can be separated scientifically. The present state of EQ predictability can be briefly formulated as follows (Gusev, 1998): "EQ's are unpredictable in the framework of present paradigm". Kuhn (1962) introduced entity "paradigm". as a system of rules and standards of scientific activity and system of ideas and laws considered as the truth by scientific community for a certain amount of time for a certain science. After the acceptance of a paradigm, a stage of "normal science" follows. Accepted theories are used to solve the problems that can be described in the framework of the given paradigm, but the nature is always more complicated than any of our theoretical models. Some anomalies emerge which cannot be explained in terms of accepted paradigm. When the quantity of anomalies reaches a critical value, scientific revolution occurs which results in the change of paradigm. It is not yet clear how fundamental the changes of paradigm for explanation of the anomalies must be connected with EQ problem. For an explicit description of principal processes involved in the phenomenon of EQ preparation and precursor formation, it may be necessary to change the paradigm in several stages:

1. Elaboration of new more adequate models of geological environment using the laws of modern physics only.

2. Laws of today's physics turn out to be insufficient to explain all phenomena connected with EQ and some extension of physics by means of introduction of new entities and laws will be needed. A review of non-traditional physical conceptions and analysis of some anomalous geophysical phenomena give a ground for supposition that some subtle field(s), yet not identified in physics, is operating in the nature (Rokityansky, 1999a). One of the candidates for such subtle field can be a gravitorsional (GT) field (vortex gravity). It can be related to the gravitational field as the magnetic field is related to the electric field, i.e. by Maxwell type equations. Such an approach has been developed by Heaviside (1893), Krylov and Sobolev (1994), Klyushin (1995), Dyatlov (1996) and others. The last author introduced the possibility of mutual transformation of electromagnetic (EM) field into GT field and back. The law of GT field propagation differs from that for EM field. Then, GT field can provide a non-traditional channel of signal transport including EM precursors. It can also be supposed that the rocks in (sub)critical state (before and during fracture) can irradiate both EM and GT fields.

3. Paradigm changes affect fundamental principles of the science, for example, by means of introduction in it some spiritual and ethical categories.

It seems reasonable to try at first to use all the possibilities of present physical paradigm and, if 
all the attempts would fail, go to the next stage. This article will be limited by the first stage. Below, three statements will be formulated. The first is general, not very new and nowadays almost obvious. The second is also rather general and it implies many versions of realization that can be seen from a short review of a few recent experimental studies. The third is more concrete and less general, which will be considered in more details.

\section{Statement 1 : Earthquake preparation and formation processes of precursors take place in a much larger volume than the source focal volume.}

The attempts to explain long distance precursory phenomena by considering the processes only in focal volume usually lead to the conclusions like the following:

"The critical analysis of the proposed theoretical models of the seismic-related variations cf electromagnetic field in the ULF frequency range has demonstrated that none of the theories can be fully adopted because of the essential intrinsic difficulties" (Surkov and Pilipenko, 1999). In Molchanov et al. (1994), the possibility of precursors' generation out of focal volume is allowed (although yet not used):

"The generation of (EM) emissions is not well understood at present and only qualitative speculations are used for some explanation of the observational results. There is no conventional theory; neither about what the nature of the source is, nor where it is placed (inside the ground medium, near the ground surface, in the atmosphere or in the ionosphere)."

Nowadays, abundant observational data and modeling validate the first paradigm: "The area where premonitory phenomena are formed is much larger than the source of a subsequent large earthquake" (Keilis-Borok et al., 1999). To be consistent, it is reasonable to suppose that the process of EQ preparation itself should not be limited by the source volume. It is obviously the case for earthquakes connected with movements of plates and for magma emplacement mechanism described below. So, the first paradigm can be accepted in the form given as the title of this chapter.

\section{Statement 2 : Volatile transport is one of basic features of earthquake preparation and precursors' formation processes.}

In common, this statement is almost obvious because magma and volatile are the most mobile and active components of the Earth's crust and upper mantle. But the study of its content and essence is yet in very beginning. A few approaches will be reviewed briefly, some of which can be incorporated into a more specific paradigm.

\subsection{Diffusion model}

Gufeld et al. (1999) supposed that degassing of the Earth is one of main factors controlling seismicity and processes of energy transfer in the lithosphere. Their model incorporates the effect of ascending diffusion of helium, hydrogen and possible other gases on the crystalline structure of rocks. The model is based on laboratory experiments with olivine, pyroxene and quartzite. Activation, i.e. implantation of helium or hydrogen into crystalline structure of the rocks was performed by heating during a few hours up to transition of the rock to a metastable state that was controlled by structural analysis (X-ray diffractometer) which proved a strong change in the rock's microstructure. Gas liberation from activated rocks (controlled by mass spectrometry) turned out to be non-monotonous. Faster degassing occurs from pores and hidden micropores, slower one from deep pores and crystal defects with time constant variation 
from minutes to months. The biggest changes in host rocks structure take place for elements with variable valence, such as Fe in the structures of olivine and pyroxene. Authors calculated the pore helium pressure for concentration inherent for the continental crust and made conclusion that this pressure is sufficient for structural recovery of the rock (if it was microfractured by previous EQ) and for several percent volume change. Gufeld et al. (1999) supposed that these effects under favorable conditions can lead to an earthquake because gas implantation from deep interior into a lithosphere structure can result in volume and compaction changes, growth of tensile stress, creep. Spreading of the gas to next structures can give "relay-race" process of energy transfer which can include large horizontal component depending of gas penetrability of lithosphere.

\subsection{Percolation model}

Iudin and Kas'yanov (1999) proposed a percolation model of seismic activity. The lithospheric substratum is considered as two-phase system: supersaturated solid solution and a gas emanating from it. Growth of gas volume results in connectedness of intraporous space and forming a cluster of light phase. Deficit of gravitational force applied to the light phase (positive buoyancy) create local stress and when it exceeds (as a result of gas volume growth) the durability of the firm phase (percolation threshold) an EQ occur. A very slow process of degassing leads to structural changes of rocks predetermining a number of precursors and $E Q$.

\subsection{Model of hydrogen emanation}

Freund et al. (1999) developed a new concept according to which most of rock forming minerals composing lithosphere can emanate molecular hydrogen as a result of fracturing. It is the case even for highly reduced environment like the lower crust and upper mantle, because even nominally anhydrous minerals must contain some water as "impurity" $\mathrm{OH}^{-}$in their crystal structures. Essential part of the "impurity" exist as $\mathrm{OH}^{-}$pairs forming a link $\mathrm{X} / \mathrm{OH}-\mathrm{HO} / \mathrm{Y}$, where $\mathrm{X}, \mathrm{Y}=\mathrm{Si}^{4+}, \mathrm{Al}^{3+}, \mathrm{Fe}^{3+}, \mathrm{Mg}^{2+}$ etc.(in many minerals $\mathrm{X}=\mathrm{Y}$ ). Normally the igneous rocks are very good insulators. Freund and Borucki (1999) struck the cores of the igneous rocks by a steel ball with low velocity $90 \mathrm{~m} / \mathrm{s}$ and observed molecular hydrogen emanation, transient electromagnetic fields on the core and light emission during milliseconds. They explain the effects by redox conversion $\mathrm{X} / \mathrm{OH}-\mathrm{HO} / \mathrm{Y}=\mathrm{X} / \mathrm{O}-\mathrm{O} / \mathrm{Y}+\mathrm{H}_{2}$. Peroxy link $\mathrm{O}^{-}-\mathrm{O}^{-}$can be considered as defect electrons in the $\mathrm{O}^{2-}$ sublattice. The defect electrons form positive charge cloud, decay of which create transient EM phenomena (potential on the surface of the sample $0.5-1.5 \mathrm{~V}$ ) and light emission. The authors believe that the acoustic wave generated by ball impact is the cause of redox conversion and transient EM phenomena. They expect the same effects when a natural in-situ rock fracture occurs. Such a process being developed in the bulk of fracture zone can give rise large amount of hydrogen which in a time can appear in underground water and atmosphere.

\subsection{Model of ionospheric earthquake precursors}

Sorokin and Chmyrev (1999) advanced a physical formation model of ionospheric EQ precursors. Injection of gases (including radioactive elements like radon) from the lithosphere into the atmosphere is considered as a primary cause of the following chain of processes: increase of air conductivity, increase of vertical currents between earth and ionosphere, heating of ionosphere and enhancement of electric field in it. These phenomena, as authors theoretically show, lead to dissipative instability and conductivity irregularities in the lower ionosphere which can radiate ELF/VLF electromagnetic fields. Heating of lower ionosphere leads to 
vertical plasma motion and deformation of the F2 layer.

\subsection{Observation of VLF EM precursor source}

Strong support to the idea of uplifted source of VLF (1-10 kHz) EM radiation has been done in the experimental study by Fujinawa et al. (1999). Impulses of the VLF radiation were recorded in four stations with time resolution of $1 \mu \mathrm{s}$ that gives a possibility to define arrival time of the impulses at each station with error less than $10 \mu \mathrm{s}$ and find the location of VLF source with accuracy of a few tens of $\mathrm{km}$. During the monitoring interval, two inland EQs (M 5.8 and 5.0) in the central Japan occurred. Two days before (and after) EQs, strong enhancement of VLF pulse number was observed, sources of which were widely distributed over broad area of the Pacific ocean at distance $100-500 \mathrm{~km}$ from the epicenter. Then, the VLF source was not underground, but several tens of kilometers above the ground surface, and from several tens to several hundred kilometers away from the epicenter. Fujinawa et al. (1999) supposed that the VLF waves are intrinsically related to the earthquake preparation process, and are generated through injection of aerosols and radioactive gases into the atmosphere and by electrostatic field change.

The review can be continued but it cannot lead now to the closed concrete paradigm. Many new studies should be done to achieve the goal. Now, before substantiating the third statement, short description of seismic electric signal (SES) will be done, because SES can be treated as the best studied precursor both spatially and temporally.

\section{Seismic Electric Signals (SES)}

In the early $80 \mathrm{~s}$ Prof. P. Varotsos, K. Alexopoulos and K. Nomicos (VAN-group, VAN-method of EQ-prediction) discovered in Greece special EQ precursors which they named seismic electric signal (SES) (Varotsos et al., 1981, 1993, 1996). They created an effective system of field measurements of electric field consisting of several dipoles with different lengths. Careful analysis of the records produces a basis for separation of SES from other magnetotelluric and cultural noise. Reliability of the separation has been checked by several independent groups of experts who visited Greece (Nagao et al., 1996, Uyeshima et al., 1998). According to VAN group almost every SES turns out to be followed by a relatively strong EQ that gives ground to use SES for EQ prediction. Such attempts meet strong critics (Geller, 1997) but neutral statistical test shows that VAN results are significant (Park et al., 1996). Having continuous measurements since 1981 at 16-18 stations in Greece, VAN group made analysis of every SES events in comparison with the following EQ. So "rules of game" for VAN prediction method have been established empirically. Consider principal features of SES as they described in Varotsos et al. (1993; 1996).

F1. Duration of a SES is between half a minute and several hours.

F2. The time lag between SES and subsequent EQ varies from 7 hours to a month, but it can be longer for the strongest $E Q$.

F3. The form of a SES can be step-like or bay-like impulses. Impulses can have a gradual or abrupt (within several seconds) onset and gradual or abrupt cessation. The combination of gradual onset and abrupt cessation has never been observed.

F4. Two or more SES connected with the same seismic area and recorded at the same station occasionally have a strikingly similar form, the same polarity and polarization.

F5. For a given pair "seismic area - station" the relationship between the SES amplitude E and EQ magnitude $M$ has been found to be, 
$\log \mathrm{E}=\mathrm{a} \mathrm{M}+\mathrm{b}$

where the slope $a=0.34-0.37$ is almost constant even for different pairs, while the intercept $b$ varies widely.

F6. Selectivity effect. A given station $\mathrm{S}_{\mathrm{A}}$ situated at area $\mathrm{A}$ may be able to record SES preceding $E Q$ at seismic area $B$ but not at area $C$, even if distance $A C$ is less than $A B$. This is observed even if $A, B$ and $C$ lie on the same straight line. Selectivity is not reversible: although $S_{A}$ may be sensitive to $E Q$ from $B$, station $S_{B}$ at $B$ is not necessarily sensitive to $E Q$ from seismic area $A$.

F7.Alternativity effect. SES preceding EQ of the same epicentral zone near Kefallonia island of Ionian sea can appear or in IOA or in PIR stations (being almost in opposite directions) but not in both simultaneously (Uyeda et al., 1999).

F8.Extraordinary SES locality in the KER station.

The selectivity effect gives a possibility to predict in what seismic area the impending EQ should be expected, and SES amplitude yields estimation of EQ magnitude M.

Several theoretical models of SES phenomenon have been published (Gershenzon and Gokhberg, 1993; Lazarus, 1996; Molchanov, 1999; Slifkin, 1996; Varotsos et al., 1993 and others). All considerations operate with essentially the same model: the cause of SES is supposed to be the accumulation of strain in focal volume of future EQ. The characteristic subsurface rupture length is $5 \mathrm{~km}$ for $\mathrm{M}=5,15 \mathrm{~km}$ for $\mathrm{M}=6$ and $25 \mathrm{~km}$ for $\mathrm{M}=6.5$ (Burton, 1996), - the numbers estimate the dimension of source. Field of such small source strongly attenuate at long distances. Unipolar source fields diminish as $1 / \mathrm{r}^{2}$ in $3 \mathrm{D}$-space, as $1 / \mathrm{r}$ in $2 \mathrm{D}$-space (conducting surface or thin sheet surrounded by an insulator) and do not diminish in 1D space (linear conductor becomes an equipotential line in stationary case). An electric source, generated in focal volume of $\mathrm{EQ}$, is traditionally considered as bipolar and is treated as dipole at long distances. The field of dipole vanishes as $1 / \mathrm{r}^{3}$ in $3 \mathrm{D}$, as $1 / \mathrm{r}^{2}$ in $2 \mathrm{D}$, but electrical current does not vanish for a closed linear circuit (1D-case) in the DC approximation. Introducing a highly conductive $1 \mathrm{D}$ path, one can explain long distance propagation of electrical signal. Such approaches were developed by the VAN group last years (Varotsos et al., 1998, 2000a, b). Conductive path up to hundreds km long can be composed of intersecting system of conducting faults which are widely spread in the Earth crust (Ingerov et al., 1999).

Bernard (1992) supposed that stress from focal volume can be transmitted hydraulicly via long distance to create stream potential in the vicinity of SES sensitive place. It can be a good explanation if such long distance hydraulic paths really exist.

More difficult for explanation is the discreteness of SES and its form.

-Why does a SES appear, as a rule, only once several days or weeks before an EQ and no consequent appearances occur despite of the strain in the source increases more and more?

-Why have long lasting SES ("electrical activity") almost constant amplitude of impulses?

-Why does almost every SES be followed by an EQ?

It looks like some kind of informational signal, like nervous impulses carrying address of the muscle and command what to do, and after short delay for preparation the muscle executes the command. Such comparison can pull us up to the third stage of paradigmal changes but it is outside of the scope of the article. In any case the questions and features mentioned above lead us to the suggestion: SES are generated not in the focal zone of a future EQ.

\section{Statement 3 : Magma emplacement and transport along the Earth's crust can be the cause of earthquakes and their precursors}


Crustal intrusions are widely spread in the Earth's crust. Near some of them embedding rocks' ruptures are observed that indicates EQ occurrence during intrusive activity. Guterman and Khazan (1994) supposed that present-day crustal seismic activity or some part of it can be a manifestation of contemporaneous magma intrusion activity. Their basic model consists of mantle and crustal magma chambers and a channel connecting them. In Rokityansky (1999b) this approach was supplemented by consideration of lateral magma transport and applied for the explanation of some peculiarities of seismic electric signal (SES). Below an improved and extended version of the consideration is presented in more details accounting for recent works.

\subsection{Radiating dyke swarms as manifestation of long distance magma transport}

Extensive isotopic, geochemical, structural and geophysical studies of igneous rocks (dykes, sills, magma fields in continental igneous provinces and oceanic plateaus) as well as surveys on the Venus give evidence that long distance sub-horizontal magma transport is a universal phenomenon (Ernst et al., 1995; Cashman et al., 1998; Elliot et al., 1999). Mafic dyke swarms are the concentration of dykes (inclined sheets, sills, magma fields) which usually show radial or fanning pattern radiating from a center. Textural and magnetic fabric studies, isotope and geochemical analysis show that major, trace and isotopic composition of laves is very uniform along the radius of a swarm, and is clearly distinct from the composition of laves in other swarms. It gives evidence that all magmas of a (giant) radiating dyke swarm have one common source, - homogeneous central magma chamber (batch, reservoir), and proves long distance lateral magma transport in the lithosphere.

The largest dyke swarm was found in Canadian shield, and it is the Mackenzie giant radiating dyke swarm with radius up to $2800 \mathrm{~km}$ long (Fig. 1). At least 60 dyke swarms have been identified in the Canadian Shield. As it is commonly accepted, the most shield areas are underlain by lithospheric mantle roots since the Archean. These roots are chemically and tectonically not a reasonable magma source for the abundant Proterozoic dyke swarms that traverse the shields. Nor would the magma likely have originated beneath the $200 \mathrm{~km}$ thick mantle root and come through it easily. The problem can be resolved by a model (Ernst et al., 1995), in which magmas injected from mantle plume have ascended vertically to a shallow level and then propagated laterally for great distances away from the plume into craton. The "shallow level" is apparently defined by a neutral buoyancy condition. The mafic magma usually has a higher density than the upper part of relatively light continental crust that creates the possibility of long distance lateral underground intrusion (Fig. 2).

Large dyke swarms related to mantle plumes are typically associated with divergent plate margins, smaller swarms (tens to few hundreds of kilometers long) are associated with volcanic edifices and large intrusions. Recent and coeval dyke swarm formation occurs and can be observed at volcanoes. Gudmundsson et al. (1999) studied more than 5000 dykes of late Tertiary to Holocene age in Iceland and Tenerife, Canary Islands. They found that most dykes become arrested at various crustal levels and never reach the surface to feed eruptions. They concluded that during periods of volcanic unrest the probability of volcanic eruption is only a small fraction of probability of dyke injection from magma chamber.

\subsection{Manifestations of the magma movement in volcanic regions}

The modern volcanic radial intrusions are studied in Iceland and Hawaii (Ernst et al., 1995). Detailed seismic studies on volcano Kilauea, Hawaii, revealed central shallow magma chamber at 2-6 km depth. Depth interval 2-4 km corresponds here to neutral buoyancy condition. From time to time magma from this central reservoir is injected laterally along the 


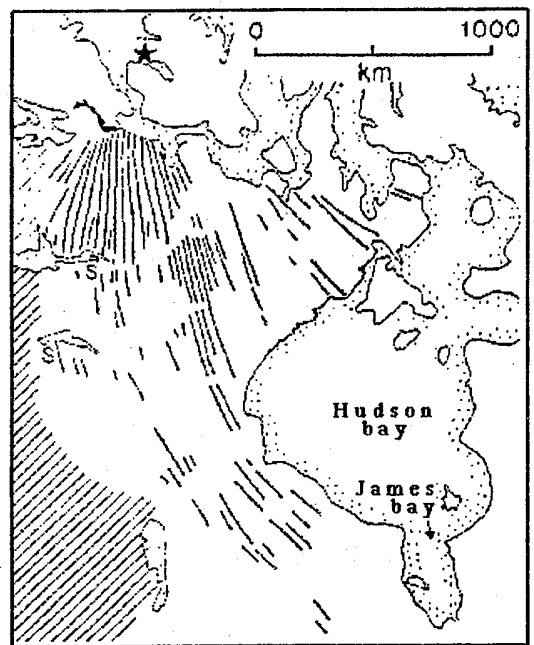

Fig. 1 Mackenzie giant radiating dyke swarm of Proterozoic age in Northwest Canada (thick lines). Star marks the focal zone of the swarm. Aquatic areas and Phanerozoic sediments (diagonal ruled pattern in the western part of the territory) are not available for dyke study. Small black area near foci marks coeval volcanic rocks, S - sills (Ernst et al., 1995)

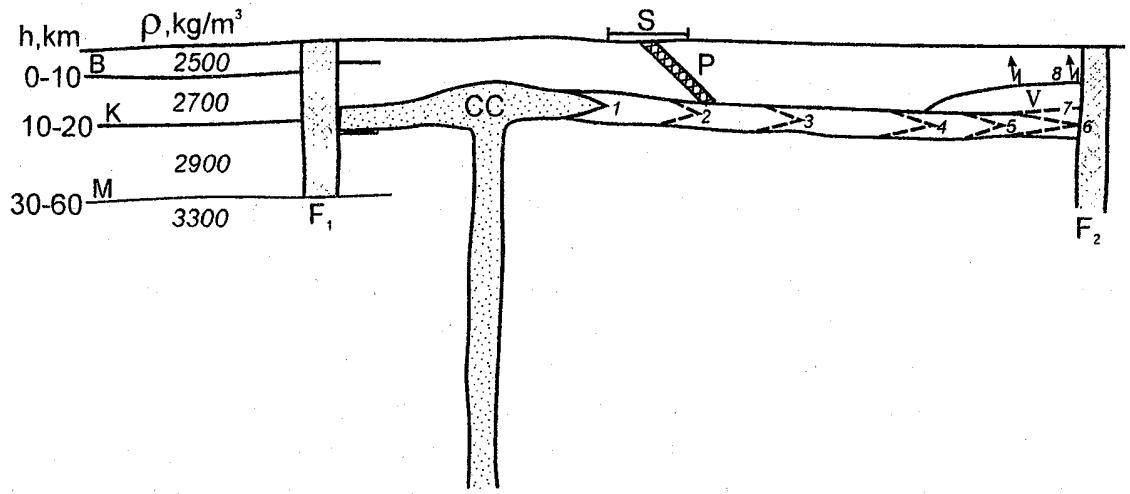

Fig. 2 Model of dyke lateral emplacement along neutral buoyancy level in the Earth's crust. Symbols used are: $M C$, mantle chamber; $C C$, crustal chamber; $F_{1}$ and $F_{2}$, zones not penetrable for magma emplacement; $\mathrm{P}$, a path for a precursor propagation to the Earth surface; $\mathrm{S}$, area sensitive for a precursor.

Generalized density model of the Earth's crust is given in the upper left part: B, crystalline basement surface; K, Konrad boundary; $M$ - Moho boundary. In the right part: 1 - 6 are consequent positions of a dyke lateral emplacement: 1 , before emplacement; 6 , arrest of the dyke emplacement. 6 - 8 are magma accumulation in local volume and stress growth in adjacent crustal rocks. 8 - Earthquake (zigzag arrow) when the stress exceeds durability of host rocks. V is source focal volume (i.e. volume where seismic waves are generated during given EQ) plus magma body adjacent to it. 
shallow neutral buoyancy horizon into one of radiating rift zones up to $120 \mathrm{~km}$ long. Recent "volcanic crises" there occurred on February 1, 1996 (Ohminato et al., 1998). During 4.5 hours the summit caldera (Fig. 3) inflated, and then 3 days deflated to normal state that has been recorded by tiltmeter (Figs. 4a, b). During the rapid inflation phase, ground displacements of sawtooth form (Fig. 4c) and pulses of ground velocity (Fig. 4d) have been recorded by seismic stations (Fig. 3a). Put together with other field observations, these data strongly suggest that this activity is the result of forceful magma injection beneath Kilauea volcano. Detailed inversion of seismic data (Figs. 3a, b) yields a crack-like source acting as a buffer to the flow of magma into the east rift (Figs. 3c, d). Rapid inflation in the buffer during 1-3 minutes (stress accumulation), was followed by more rapid deflation during 5-10 s (magma and/or gas injection and stress release) as it is seen in Fig. 4c. Such rapid powerful injections undoubtedly must be accompanied by generation of electric signals of approximately same waveform.

Analogous phenomena were observed on several volcanoes, for example at the Soufriere Hills Volcano, Montserrat island, during periodic dome growth and collapse in 1996-1997 (Voight et al., 1998). Dome inflations-deflations with periods from 8 to 25 hours correlated with seismicity, explosions and pyroclastic flow activity. Pulse regime of magma flow was explained by two competing processes: 1) Exsolution and degassing from uppermost part of magma chamber increases melt viscosity and thus inhibits magma flow. Volatile loss causes microlite crystallization. As microlites become abundant they increase the bulk viscosity and strength by particle-to-particle interactions. The resulting plug of stuff magma retards the flow of less viscous volatile rich magma from deeper levels. 2) Pressurization of the magma beneath the degassing plug which works to rise the plug and overlying dome. Eventually the pressure reaches a threshold sufficient to push the degassed plug out of conduit, along with an accompanying portion of magma richer in volatiles. This extrusion relaxes pressure, causing deflation of the volcano. Rapid decrease in pressure also promotes degassing and microlite nucleation in fresh magma, and new cycle begins.

Amplitude modulation of magnetic field at the frequency $223 \mathrm{~Hz}$ recorded on Unzen volcano in Japan two months prior to the dome formation is shown in Fig. 5 (Hata et al., 1998). Intermittent oscillations with period approximately 1 min manifest some mechanical movements connected with magma emplacement.

An example of SES activity recorded in Greece is presented in Fig. 6. Comparing the last three figures, one should keep in mind that signals on volcanoes were observed at the distance of order $1-2 \mathrm{~km}$ from the source while distance to SES source is not known and may be much larger. Some similarity of transient signals of magmatic origin and SES is seen in Figs. 4-6.

\subsection{Rate of magma emplacement.}

At Krafla in Iceland, and often at Kilauea in Hawaii, intrusion begins with subsidence of the caldera region and downrift migration of a swarm of earthquakes at some tens of centimeters per second. The migration is interpreted as the propagation of the dike at a depth (Rubin, 1995). The same velocity of swarm migration was observed during initial phase of the 2000 Miyake-Kozu swarm event (Japan Meteorological Agency, 2000). The velocity of swarm migration gives lower limit of magma emplacement rate.

Magma emplacement process depends on the competition between the rate at which the dike thickness increases after its opening due to magma propagation and the rate at which the aperture for flow decreases due to solidification. Dissipation of energy from hot magma into cold embedding rocks put strict limitation on the duration of magma emplacement process. Energy sources available to retard solidification include an excess magma temperature above 

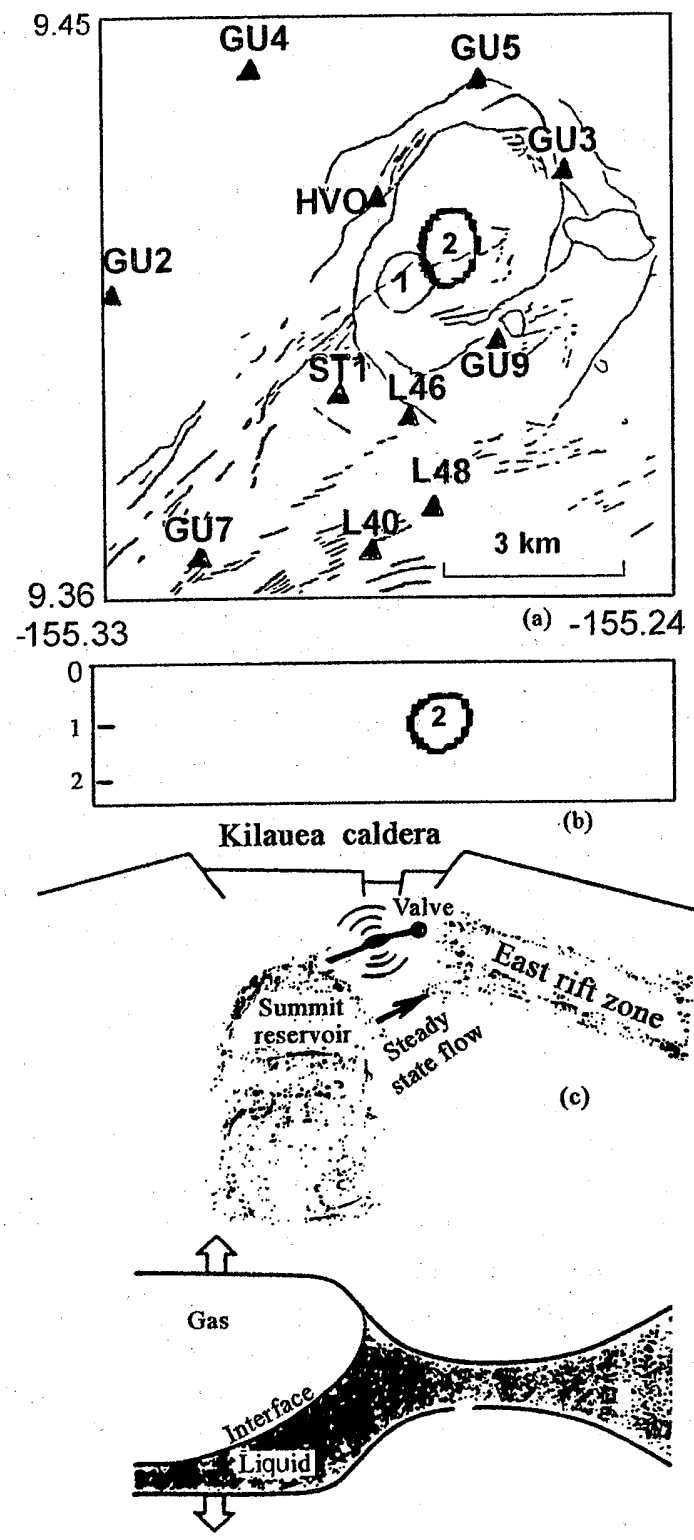

(d)

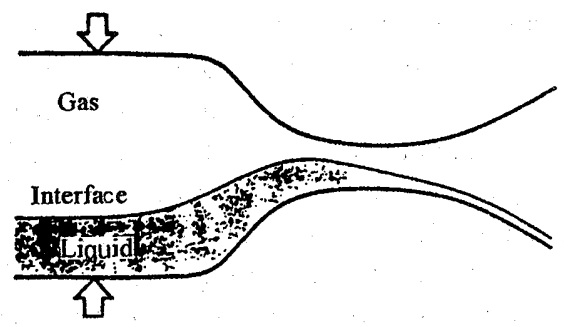

Fig. 3 "Volcanic crisis" of February 1,1996 on Kilauea Volcano, Hawaii. (a) Map: seismic stations (triangles), caldera (1) and source focal volume according to inversion of seismic data (2). (b) Cross section: ( 2 as in a). (c-d) Models of magma transport (Ohminato et al., 1998). 


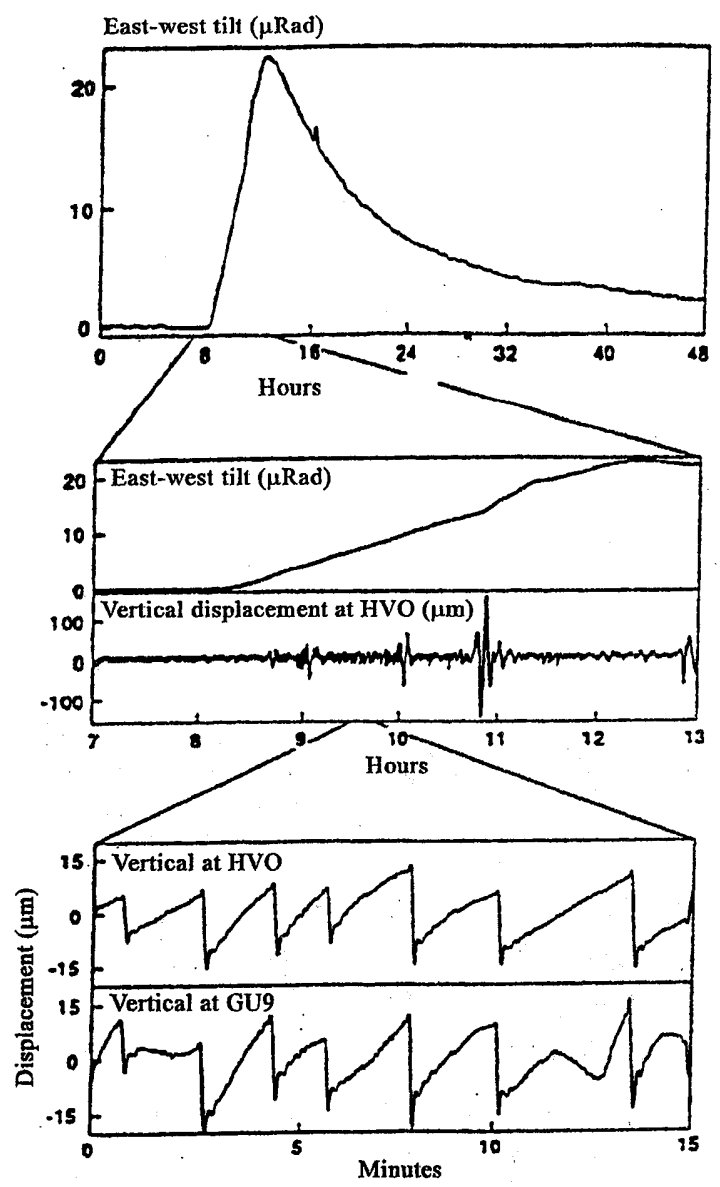

(a)

(b)

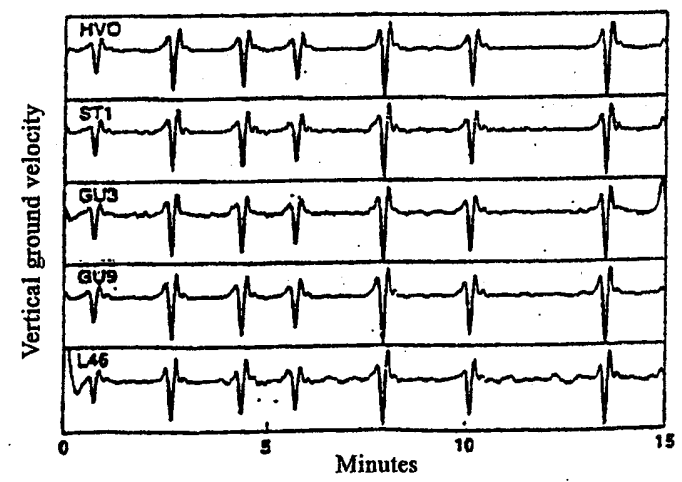

(c)

(d)

Fig.4 Records on Kilauea at February 1, 1996. (a) Tilt near HVO station 1-2 February. (b) Tilt and ground displacement during inflation phase. (c) Sawtooth displacement waveforms during active phase at two stations. (d) Ground velocity for the same interval at five stations

(Ohminato et al., 1998) 


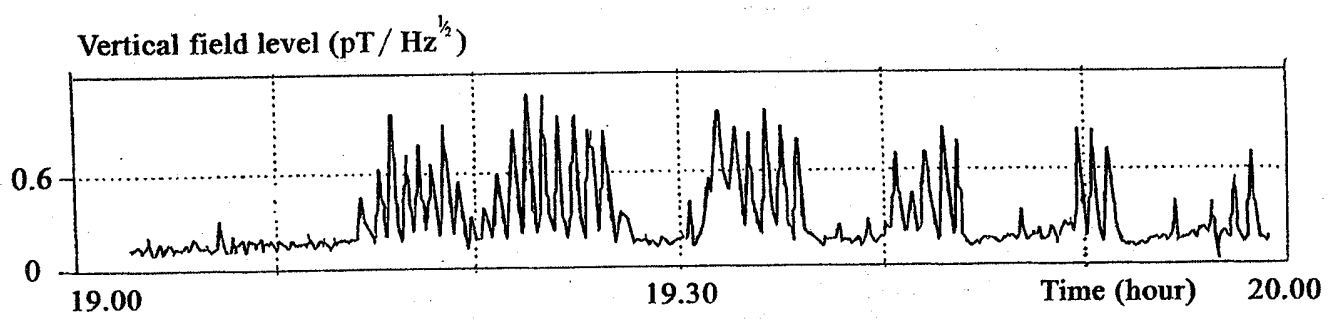

Fig. 5 Long period amplitude modulation of magnetic field oscillations at $223 \mathrm{~Hz}$ near Unzen volcano on January 4, 1993 (Hata et al., 1998)

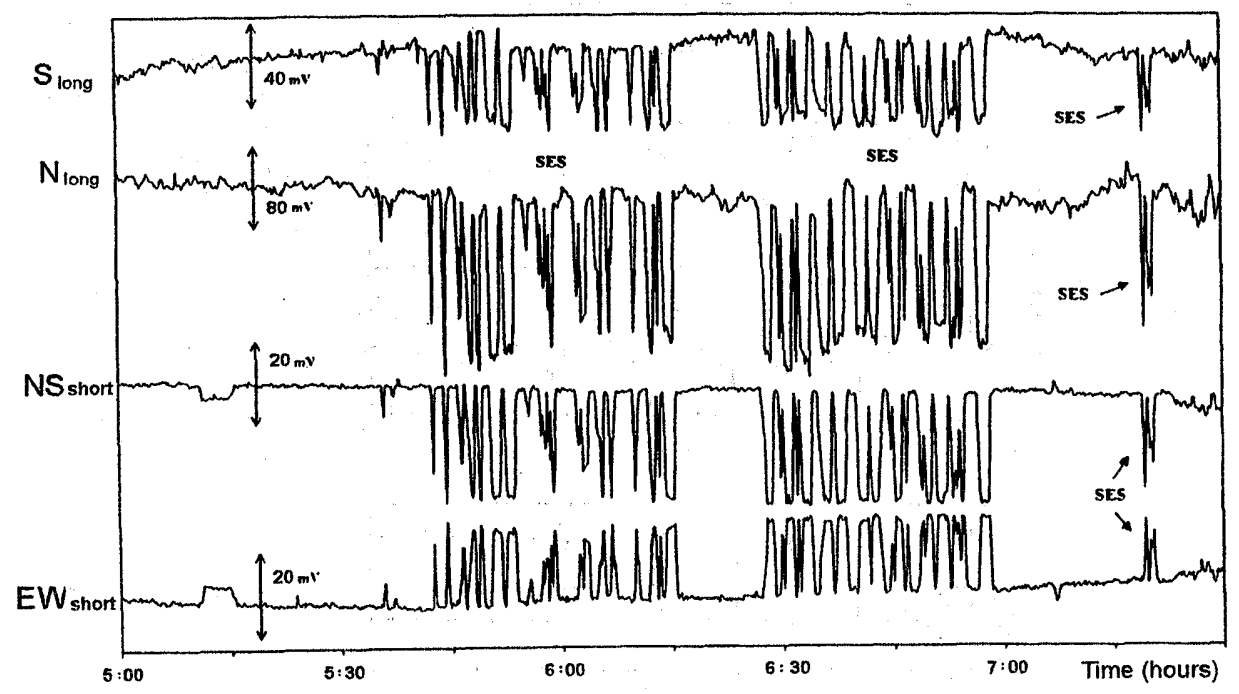

Fig.6 Example of SES activity at five channels of Lamia station in Greece on October 10, 1999 (data of P.Varotsos) 
solidus, release of latent heat during crystallization, and viscous dissipation (Fujii and Uyeda, 1974). Viscous dissipation can compensate the conductive heat loss that create the conditions for very long distance magma transport.

The mechanism of lateral magma emplacement is the subject of intensive theoretical and model studies, see for example Rubin (1995), Fialko and Rubin (1998, 1999). Preliminary rough calculations (Ernst et al., 1995, p.44) yield the estimation of the velocity of magma flow be equal to approximately $1 \mathrm{~m} / \mathrm{s}$ for long (order $1000 \mathrm{~km}$ ) flow and $10 \mathrm{~m} / \mathrm{s}$ for short (order 30 $\mathrm{km}$ ) flow for the same driving pressure $30 \mathrm{MPa}$ and mean dyke width $25 \mathrm{~m}$. It gives the total time of dyke emplacement varying from several hours to several weeks. Taking into account an additional time for stress accumulation in the focal volume, the coincidence with time lag between seismic electric signal (SES) and EQ ranging from 7 hours to a month (Varotsos et al., $1993 ; 1996)$ is rather good. If the coincidence is not accidental and the connection of the phenomena involved really exists, it suggests that SES is the manifestation of the beginning or some essential phase of magma emplacement, which can be at long distance from focal volume of future EQ.

\section{A possible scenario of magmatic origin earthquake preparation}

During seismic quiescence, mantle magma chamber MC (Fig. 2) gradually accumulates high extra pressure. The upper surface of the chamber elevates slightly giving rise to long term precursors formation which can be observed over large area above and around of mantle chamber. The high pressure of mantle chamber then opens a path upward and hot, volatile rich magma elevates up to the level of neutral buoyancy. The density of the rocks in the Earth' crust usually grows with depth stepwise at the surface of basement, and at the Konrad and Moho discontinuities (Fig. 2). Magma density depends on its content and physical conditions. Thus, the level of neutral buoyancy can coincide with different discontinuities in different environments. Crustal magma chamber $\mathrm{CC}$ formation occurs at that level. When excessive pressure in $\mathrm{CC}$ accumulates, lateral magma emplacement can begin under favorable stress conditions in surrounding host rocks.

\subsection{Excessive pressure in magma channel}

$\mathrm{F}_{2}$ in Fig. 2 presents a barrier which arrests the magma emplacement process. After that, the excessive pressure is not released by means of lateral magma emplacement and can create stress accumulation in any place along the lateral magma flow or around CC.

We can suppose that the excessive pressure inside magma channel is approximately the same along all magma paths, because hot magma in the axial part of the channel conserves during a time (dependent of channel width) the properties of liquid with low viscosity (for the mafic magma). Meanwhile, the durability of marginal parts ("walls") of magma channel can be supposed to be higher for the longer time passed after the channel formation. It can be explained by interaction between hot magma and cold host rocks that results in solidification. After channel opening, volatile magma penetrates the pores of host rocks, creates metamorphic transformations and finalizes by crystallization in marginal parts of the channel. The longer this process lasts, the thicker and more durable channel's walls it forms. It means that the most vulnerable part of the magma channel can be expected in the remote part of the channel where walls are the youngest one. In Fig. 2, the stress accumulation is shown in the vicinity of the barrier $\mathrm{F}_{2}$ : positions 7 and 8 . 


\subsection{Conditions for the preparation of a strong earthquake caused by magma emplacement}

After the arrest of lateral magma emplacement by barrier $F_{2}$, volatile magma tries to find other paths and fills some additional volume(s) around the channel (volume 7-8 in Fig. 2) up to the moment when all possible paths will be arrested (alternatively magma emplacement would continue).

The larger a magma body is, the longer is the time of its formation (time of magma flow into the volume). As it is well known (Burton, 1996), the larger the magnitude of EQ is, the larger its source focal volume is. Assuming proportionality (or any direct correlation) between magma body volume and source focal volume, one can see that the larger the magnitude of EQ is, the longer the time lag between lateral magma emplacement and consequent $\mathrm{EQ}$ is. Analogous correlation was repeatedly reported by VAN group: for the larger magnitude of EQ, we expect the longer time lag between related SES and the EQ (Varotsos et al., 1993, 1996). Excessive pressure in magma channel and magma body(ies) forces deformations and stress accumulations in the surrounding host rocks. If the surrounding host medium (half-space) is uniform and ideally elastic, magma accumulation in a body can be compensated by Earth's surface elevation. It was proved analytically for a thin circular horizontal body (Fialko et al., 2001). Stress accumulation does not occur in this case (except local zones near the places where body's surface has high curvature) and therefore conditions for strong EQ preparation do not arise despite of strong geodetic anomaly.

Real Earth's crust supports large surface loads such as mountains that require its strength to be at least $100 \mathrm{MPa}$ (Kanamori and Brodsky, 2001). Stress drop during a crustal EQ is typically of order of $10 \mathrm{MPa}$. This means that the stress in the crust is spatially very heterogeneous, and the system organizes itself into a precarious state with low stress on major faults (Kanamori and Brodsky, 2001).

Excessive pressure carried by magma is normal to its surface and therefore it can initially create only tensile stress in the adjacent host rocks. But the complexity of geological medium always transforms any pure component (shear, tensile or isotropic force system ) into a superposition of all components (Julian et al., 1998). It is proved by an analysis of moment tensor of real earthquakes which usually have composite structure, i.e. besides double-couple component (corresponding shear stress release), compensated linear vector dipole (corresponding tensile stress) and volumetric isotropic components are also presented. Two latter components have comparable, for some earthquakes dominant, value comparatively to the first one. For example, compensated linear vector dipole mechanism was found dominant for four crustal EQs with magnitude larger than 6 occurred in Long Valley caldera, California, in May 1984 (Miller et al., 1998). Therefore, data of moment tensor analysis do not contradict to magmatic origin of strong crustal EQs and in many cases support it.

So, the common conditions for strong EQ preparation are adequate durability (strength) distribution of the host rocks and sufficient energy of intruded magma to create large scale stress accumulation up to failure threshold.

\section{Formation of a precursor in the magma emplacement model}

Opening of crack at the side tip of CC (Fig. 2) in the process of magma emplacement should be accompanied by mechanical (microseismisity or forshocks), electrical (SES) and other signals. The possibility of their registration depends on many factors:

- intensity of source which is located in the place of crack formation and moves in the course of magma emplacement. This movement is shown in Fig. 2 by consequent positions $1-6$ of 
magma emplacement tip.

- distance to observatory,

- physical properties of the rocks between source and observatory.

For seismic electric signals (SES), strong attenuation both geometrical and dissipative is expected based on theoretical estimation for homogeneous and horizontally layered conductivity structures (Bernard, 1992). Meanwhile, SES in Greece have been observed at the distance up to $500 \mathrm{~km}$ from the related EQ (Varotsos et al., 1993,1996).

In the magma emplacement model, an observatory at long distance from the related EQ can be rather near the place of crack formation (tip of propagating magma emplacement), where the source of a precursor is assumed to be located.

\subsection{Favorable path for precursor propagation}

The conductivity distribution in the upper crust is heterogeneous. Deep faults, being better conductors than embedding rocks, sometimes form continuous intersecting systems. It is reasonable to suggest that in the Earth's crust, some well conducting channels do exist along which SES can effectively "propagate" to a "sensitive" area on the Earth's surface (Varotsos et al., 1998, 2000a, b). In Fig. 2 conducting path $\mathrm{P}$ is drawn near the position 2 of magma emplacement. Let us suppose that SES formation occurs in all stages of the emplacement but it can arrive effectively at the Earth's surface only when SES source (tip of magma emplacement) passes near the position 2 when SES propagation can use the path P. It explains why SES appears only once before EQ and only in limited "sensitive" area.

Alternatively it can be assumed that strong SES is generated only once during the first opening of the lateral magma channel (position 1 in Fig. 2). Alternative interpretation of path $P$ can be a hydraulic channel transferring pressure to the vicinity of sensitive place where electrokinetic phenomena convert it into electric signal (Bernard, 1992).

\section{Conclusion}

Magma emplacement process can be accompanied by a variety of small or moderate EQs of magmatic origin which form usually a swarm. If several interstitial moderate scale magma reservoirs would be formed along the magma path, they can generate a sequence of stronger EQs, which can be treated as forshocks of the following main shock. All these precursory EQs tend to migrate along the principal magma path. Exactly such a behavior was observed recently in Japan during the initial part of 2000 Miyake-Kozu swarm event. Earthquake swarm started at Miyakejima on June 26 and migrated about $25 \mathrm{~km}$ to the NW direction and on July 1 a strong EQ of $M=6.4$ occurred at NW end near the Kozu Island (Japan Meteorological agency, 2000; Sakai et al., 2001).

\section{Acknowledgements}

The author thanks Profs. S.K.Park and N.Fujii for critical reading the manuscript and thoughtful constructive comments that helped him to improve this article.

\section{References}

Bernard, P., Plausibility of long distance electrotelluric precursors to earthquake, J. Geophys. Res., 97, 17531-17546, 1992.

Burton, P., Dicing with earthquakes. Geophys. Res. Lett., 23, 1379-1382, 1996. 
Cashman, K., Pinkerton, H., and Stephenson, J., Introduction to special section: Long lava flows. J. Geophys. Res., 103, No.B11, 27281-27289, 1998.

Dyatlov, A. I., personal communication, 1997.

Elliot, D. H., Fleming, T. H., Kyle, P. R., and Foland, K. A., Long-distance transport of magmas in the Jurassic Ferrar Large Igneous Province, Antarctica. Earth Planet. Sci. Lett., 167, 89-104, 1999.

Ernst, R. E., Head, J. W., Parfitt, E., Grosfils, E., and Wilson, L., Giant radiating dyke swarms on Earth and Venus. Earth-Sci. Rev., 39, 1-58, 1995.

Fialko, Y. A., and Rubin, A. M., Thermodynamics of lateral dike propagation: Implications for crustal accretion at slow-spreading mid-ocean ridges. J. Geophys. Res., 103, 2501-2514, 1998.

Fialko, Y. A., and Rubin, A. M., Thermal and mechanical aspects of magma emplacement in giant dike swarms. J. Geophys. Res., 104, 23033-23049, 1999.

Fialko, Y., Khazan, Y., and Simons, M., Deformations due to a pressurized horizontal circular crack in an elastic half-space, with applications to volcano geodesy. Part 1. Theory. Geophys. J. Int., 146, 181-190, 2001.

Freund, F., Borucki, J. G., Charge carrier generation and charge cloud propagation following $100 \mathrm{~m} / \mathrm{s}$ impacts on igneous rocks. In: Hayakawa, M. (Ed.), Atmospheric and ionospheric electromagnetic phenomena associated with earthquakes. Terra Sci. Publ. Comp., Tokyo, pp. 839-857, 1999.

Freund, F., Gupta, A., Butow, S. J., and Tenn, S., Molecular hydrogen and dormant charge carriers in minerals and rocks. In: Hayakawa, M. (Ed.), Atmospheric and ionospheric electromagnetic phenomena associated with earthquakes. Terra Sci. Publ. Comp., Tokyo, pp. 859-871, 1999.

Fujii, N., and Uyeda, S., Thermal instabilities during flow of magma in volcanic conduits. J. Geophys. Res., 79, 3367-3369, 1974.

Geller, R. J., Eartquake prediction: a critical review. Geophys. J. Int., 131, 425-440, 1997.

Gudmundsson, A., Marinoni, and L.B., Marty, J., Injection and arrest of dykes: implication for volcanic hazards. J.Volcanol.Geotherm.Res. 88, 1-13, 1999.

Gufeld, I. L., Gusev, G. A., Lyutikov, R. A., Matveeva, M. I., Seismic process as phase instability of the lithosphere. In: Hayakawa, M. (Ed.), Atmospheric and ionospheric electromagnetic phenomena associated with earthquakes. Terra Sci. Publ. Comp., Tokyo, pp. 885-909, 1999.

Gusev, A., EQS, 79; p. 71, 10 Feb.1998; p. 373, 4 Aug.1998.

Guterman, V. G. and Khazan, Y. M., A possible mechanism of strong shallow earthquake preparation.Geophys. J., 16, No.1, 22-29 (in Russian), 1994.

Hata, M., Takumi, I., Yabashi, and S., Tian, X., An anomaly of ELF band vertical magnetic flux as a precursor of dome formation at Unzen volcano and its model analysis. Phys. Earth Planet. Inter., 105, 271-277, 1997.

Hayakawa, M. (Ed.), Atmospheric and ionospheric electromagnetic phenomena associated with earthquakes. Terra Sci. Publ. Comp., Tokyo, 996 pp, 1999.

Hayakawa, M., and Y. Fujinawa, Y. (Eds.), Electromagnetic phenomena related to earthquake prediction. Terra Sci. Publ. Comp., Tokyo, 677 pp, 1994.

Heaviside, O. A., Gravitational and electromagnetic analogy. The Electrican, pp. 281-282 and $359,1983$.

Ingerov, A. I., Rokityansky, I. I., Tregubenko V. I., Forty years of MT studies in the Ukraine. EPS, 51, 1127-1134, 1999. 
Iudin, D. I., Kas'yanov, D. A., Percolation model of seismic activity. In Hayakawa, M.(Ed.), Atmospheric and ionospheric electromagnetic phenomena associated with earthquakes.

Terra Sci. Publ. Comp., Tokyo, pp. 911-917, 1999.

Japan Meteorological Agency, Recent seismic activity in the Miyakejima and Niijima-Kozushima region, Japan - the largest earthquake swarm ever recorded. Earth Planets Space, 52(8), i-viii, 2000.

Julian, B.R., Miller, A.D., and Foulger, G.R., Non-double-couple earthquakes . 1. Theory. Reviews of Geophysics, 36, 525-549, 1998.

Kanamori, H., and Brodsky, E.E., The physics of earthquakes. Physics Today, No. 6, 34-40, 2001.

Keilis-Borok, V., Kossobokov, V., Rotwain, I., and Soloviev, A., Earthquake prediction: recent results and what comes next. Abstracts of IUGG99, vol. A, p. 137, Birmingham, 19-31 July 1999.

Klyushin, J. G., Maxwell approach in the description of gravity. In: New ideas in natural sciences, RAS, St.-Petersburg, pp. 242-275, 1995.

Krylov, S. M., and Sobolev, G. A., On ultra low frequency vortex gravitational field on the Earth's surface. Dokl. RAN, 339, 396-400 (in Russian), 1994.

Kuhn, T. S., The Structure of Scientific Revolutions. Chicago University Press, 1962.

Lazarus, D., Physical mechanisms for generation and propagation of seismic electric signals. In: Lighthill, Sir J. (Ed.), Critical review of VAN. Earthquake prediction from seismic electric signals. World Scientific Publishing, Singapore, pp. 91-96, 1996.

Miller, A.D., Foulger, G.R., and Julian, B.R., 1998. Non-double-couple earthquakes . 2. Observations. Rev. Geophysics, 36, 551-568, 1998.

Molchanov, O. A., Fracturing as an underlying mechanism of seismic electric signals. In: Hayakawa, M. (Ed.), Atmospheric and ionospheric electromagnetic phenomena associated with earthquakes. Terra Sci. Publ. Comp., Tokyo, pp. 349-356, 1999.

Molchanov, O. A., Hayakawa, M., and Rafalsky, V. A., Penetration of electromagnetic emission from an underground seismic source into the atmosphere, ionosphere and magnetosphere. In: Hayakawa, M., Y. Fujinawa, Y. (Eds.), Electromagnetic phenomena related to earthquake prediction. Terra Sci. Publ. Comp., Tokyo, pp. 565-606, 1994.

Morozova, L.I., Cloud indicators of the geodynamics of the Earth crust. Izv. AN SSSR, Fizika Zemli, N 10, 108-112 (in Russian), 1993.

Nagao, T., Uyeshima, M., and Uyeda, S., An independent check of VAN's criteria to signal recognition. Geophys. Res. Lett., 23, 1441-1444, 1996.

Ohminato, T., Chouet, B. A., Dawson, P., and Kedar, S., Waveform inversion of very long period impulsive signals associated with magmatic injection beneath Kilauea Volcano, Hawaii. J. Geophys. Res., 103, 23839-23862, 1998.

Park, S.K., Strauss, D.j., and Aceves, R.L., Some observations about the statistical significance and physical mechanisms of the VAN method of earthquake prediction, Greece. In: 1 pp. 267-285.

Rokityansky, I. I., 1982. Geoelectromagnetic investigation of the Earth's crust and mantle. Heidelberg: Springer Verlag, Heidelberg, 381pp, 1996.

Rokityansky, I. I., Phenomenon of quasi-spontaneous globally synchronized variations of physical parameters. Phys.Chem.Earth (A), 24, 705-710, 1999a.

Rokityansky, I. I., Anomalous phenomena preceding earthquakes. Phys.Chem.Earth (A), 24, 731-734, 1999b.

Rubin, A.M., Propagation of magma filled cracks. Ann. Rev. Earth Planet. Sci., 23, 287-336, 
1995.

Sakai, S., Yamada, T., Ide, S., Mochizuki, M., Shiobara, H., Urabe, T., Hirata, N., Shinohara, M., Kanazawa, T., Nishizawa, A., Fujie, G., and Mikada, H., Magma migration from the point of view of seismic activity in the volcanism of Miyake-jima island in 2000. J. Geography, 110 (2), 145-155 (in Japanese), 2001.

Sharigin, S.A., Tenigin, B.Ya., and Pechurin, V.T., Use of anomalous behaviour of reptilians as biological earthquake precursor.1. Geophys. J., 17, N 2, 37-43 (in Russian), 1995a.

Sharigin, S.A., Tenigin, B.Ya., Pechurin, V.T., Use of anomalous behaviour of reptilians as biological earthquake precursor.2 . Geophys. J., 17, N 3, 38-44 (in Russian), 1995 b.

Slifkin, L., A dislocation model for seismic electric signals. In: Lighthill, Sir J. (Ed.), Critical review of VAN. Earthquake prediction from seismic electric signals. World Scientific Publishing, Singapore, pp. 97-104, 1996.

Sorokin, V. M., and Chmyrev, V. M., The physical model of electromagnetic and plasma response of the ionosphere on the pre-earthquake processes. In: Hayakawa, M. (Ed.), Atmospheric and ionospheric electromagnetic phenomena associated with earthquakes. Terra Sci. Publ. Comp., Tokyo, pp. 819-828, 1999.

Surkov, V., Pilipenko, V., The physics of pre-seismic electromagnetic ULF signals. In: Hayakawa, M. (Ed.), Atmospheric and ionospheric electromagnetic phenomena associated with earthquakes. Terra Sci. Publ. Comp., Tokyo, pp. 357-370, 1999.

Uyeda, S., Al-Damegh, K. S., Dologlou, E., and Nagao, T., Some relationship between VAN seismic electric signals (SES) and earthquake parameters. Tectonophysics, 304, 41-55, 1999.

Uyeshima, M., Kanda, W., Nagao, T., and Kono, Y., Directional properties of VAN's SES and ULF MT signals in Ioannina, Greece. Phys. Earth Planet Inter., 105, 153-166, 1998.

Varotsos, P., Alexopoulos, K., and Nomicos, K., Seismic electric currents. Prakt. Akad. Athenon, 56, 277-286, 1981.

Varotsos, P., Alexopoulos, K., and Lazaridou, M., Latest aspects of earthquake prediction in Greece based on seismic electric signals, 2. Tectonophysics, 224, 1-37, 1993.

Varotsos, P., Lazaridou, M., Eftaxias, K., Antonopoulos, G., Makris, J., and Kopanas, J., Short term earthquake prediction in Greece by seismic electric signals. In: Lighthill, Sir J. (Ed.), Critical review of VAN. Earthquake prediction from seismic electric signals. World Scientific Publishing, Singapore, pp. 29-61, 1996.

Varotsos, P., Sarlis, N., Lazaridou, M., and Kapiris, P., Transmission of stress induced electric signals in dielectric media. J. Appl. Physics, 83, 60-70, 1998.

Varotsos, P., Sarlis, N., and Lazaridou, M., Transmission of stress induced electric signals in dielectric media.Part 2. Acta Geophys. Polonica, 48, 141-177, 2000a.

Varotsos, P., Sarlis, N., and Skordas, E., Transmission of stress induced electric signals in dielectric media. Part 3. Acta Geophys. Polonica, 48, 263-297, $2000 \mathrm{~b}$.

Voight, B., Hoblitt, R.P., Clarke, A.B., Lockhart, A.B., Miller, A.D., Lynch, L., and McMahon, J., Remarkable cyclic ground deformation monitored in real-time on Montserrat, and its use in eruption forecasting. Geophys. Res. Lett., 25, 3405-3408, 1998.

(Received April 30, 2002; revised May 30, 2002; accepted June 10, 2002) 